\title{
Simultaneous Measurement of Belt Speed and Vibration Through Electrostatic Sensing and Data Fusion
}

\author{
Yonghui Hu, Yong Yan, Fellow, IEEE, Lijuan Wang, Xiangchen Qian, and Xiaoyu Wang
}

\begin{abstract}
Accurate and reliable measurement of belt speed and vibration is of great importance in a range of industries. This paper presents a feasibility study of using an electrostatic sensor array and signal processing algorithms for the simultaneous measurement of belt speed and vibration in an online, continuous manner. The design, implementation, and assessment of an experimental system based on this concept are presented. In comparison with existing techniques, the electrostatic sensing method has the advantages of non-contact and simultaneous measurement, low cost, simple structure, and easy installation. The characteristics of electrostatic sensors are studied through finite-element modeling using a point charge moving in the sensing zone of the electrode. The sensor array is arranged in a $2 \times 3$ matrix, with the belt running between two rows of three identical sensing elements. The three signals in a row are cross correlated for speed calculation, and the results are then fused to give a final measurement. The vibration modes of the belt are identified by fusing the normalized spectra of vertically paired sensor signals. Experiments conducted on a two-pulley belt-driven rig show that the system can measure the belt speed with a relative error within $\pm 2 \%$ over the range $2-10 \mathrm{~m} / \mathrm{s}$. More accurate and repeatable speed measurements are achieved for higher belt speeds and a shorter distance between the electrode and the belt. It is found that a stretched belt vibrates at the harmonics of the belt pass frequency and hence agrees the expected vibration characteristics.
\end{abstract}

Index Terms-Belt drive, cross correlation, data fusion, electrostatic sensor, finite-element modeling, speed measurement, vibration measurement.

\section{INTRODUCTION}

B ELT DRIVES are widely used in many industries for power transmission and material transportation. In comparison with chain, gear and other types of drives, belt drives offer many advantages to the design and functionality of a mechanical system, such as simple design, relatively low cost,

Manuscript received June 19, 2015; revised September 11, 2015; accepted October 1, 2015. Date of publication November 2, 2015; date of current version April 5, 2016. This work was supported in part by the National Natural Science Foundation of China under Grant 51375163 and Grant 61573140 and in part by the Chinese Ministry of Education under Grant B13009. The Associate Editor coordinating the review process was Dr. Subhas Mukhopadhyay. (Corresponding author: Yong Yan.)

Y. Hu, X. Qian, and X. Wang are with the School of Control and Computer Engineering, North China Electric Power University, Beijing 102206, China.

Y. Yan is with the School of Engineering and Digital Arts, University of Kent, Canterbury CT2 7NT, U.K. (e-mail: y.yan@kent.ac.uk).

L. Wang is with the School of Control and Computer Engineering, North China Electric Power University, Beijing 102206, China, and also with the School of Engineering and Digital Arts, University of Kent, Canterbury CT2 7NT, U.K.

Color versions of one or more of the figures in this paper are available online at http://ieeexplore.ieee.org.

Digital Object Identifier 10.1109/TIM.2015.2490958 smooth operation, long distance of power transmission, and ability to absorb sudden shocks or changes in loading. However, because belt drives rely on friction between the belt and pulleys to transmit torque, slippage may occur, particularly when the belt is not properly tensioned. Also, belt drives exhibit highly dynamic and complex behaviors such as transverse vibration and cyclic tension variation [1], which lead to sliding wear, noisy operation, and belt fatigue. In order for belt drives to operate efficiently, smoothly, and reliably, the belt conditions and operational performance of a mechanical system should be continuously monitored. Important belt parameters that should be monitored include wear, tension, damage, slip, rip, speed, and vibration [2]-[4].

This paper is concerned with the measurement of belt speed and vibration for online continuous monitoring and control purposes. A variety of measurement techniques based on optical, electrical, acoustic, mechanical, and magnetic principles have been developed. For belt speed measurement, the most common sensors are magnetic and optical angular encoders mounted on a pulley or wheel that rotates as the belt moves [5]. Because the performance of such contact type tachometers is affected by slippage and belt aging, non-contact type tachometers that measure the linear speed of the belt surface itself are desirable. Laser Doppler velocimeters can provide accurate and reliable speed measurement without contact with the moving surface [6], [7]. However, such instruments are relatively large in size, power thirsty, and expensive, and the use of delicate optical components limits their applicability in harsh and extreme environments. Ultrasonic and microwave speed sensors, which work on the same principle of Doppler effect, are typically lightweight and easy to install, but their accuracies are generally lower than their optical counterpart [8], [9]. A magnetic method has been proposed to measure the linear speed of a steel strip where magnetic marks are printed on the steel surface using a magnetic writer and are detected by downstream magnetoresistive sensors [10]. However, this method is unsuitable for measuring nonmagnetic strip materials due to the underlying principle of the magnetic sensing technique. A thermal method analogous to the magnetic method, but based on heater and infrared detectors, is proposed for measuring the speed of electrically conducting strips [11]. The measurement of transverse belt vibration requires non-contact sensing techniques since the axially moving belt surface prohibits installation of any contact transducers. A well-established technique for vibration measurement has been laser Doppler vibrometer working on

0018-9456 (C) 2015 IEEE. Translations and content mining are permitted for academic research only. Personal use is also permitted,

but republication/redistribution requires IEEE permission. See http://www.ieee.org/publications_standards/publications/rights/index.html for more information. 
the principle of laser interferometry [12], [13]. For the same aforementioned reasons, the optical technique is not well suited for practical industrial applications. Several types of proximity sensors, such as eddy current, ultrasonic, capacitive, and inductive sensors, are used to measure relative vibration of rotating shafts [14], but their applications to belt vibration measurement are difficult for reasons related to accuracy, durability, installation requirements, and material property of the object being measured.

In this paper, an electrostatic sensor array is used to achieve simultaneous measurement of belt speed and vibration in an online non-contact manner. Electrostatic sensors have been extensively used for characterization and measurement of particle flow in pneumatic conveying pipelines [15]-[18]. Recent applications of electrostatic sensors to gas path debris detection for health monitoring of an aeroengine have been investigated in [19] and [20]. Intra et al. [21] developed and evaluated a particulate air pollution sensor for continuous monitoring of size resolved particle number, based on unipolar corona charging and electrostatic detection of charged aerosol particles. Recent studies have been reported on the use of electrostatic sensors for the condition monitoring of mechanical machinery. Wang et al. [22] conducted experimental investigations into rotational speed measurement using electrostatic sensors and correlation signal processing techniques. A feasibility study of electrostatic sensing for strip speed measurement was carried out in [23]. This paper concentrates on the novel use of electrostatic sensor arrays in conjunction with data fusion techniques for concurrent belt speed and vibration measurement. The belt runs through a specifically designed sensor matrix that detects the electrostatic charges on the belt. Information about the belt speed and vibration is derived using embedded signal processing and data fusion algorithms [24]. This paper presents in detail the fundamental principle of the novel belt speed and vibration measurement system and the practical design and computational modeling of electrostatic sensors in addition to implementation and experimental assessment of the system.

\section{PRinciple ANd Modeling of Electrostatic SENSORS}

\section{A. Sensing Principle}

The surface of a traveling belt becomes electrostatically charged due to friction between the belt surface and the surrounding air. Because the distribution of the surface charges is nonuniform and the belt is moving longitudinally as well as transversely, a fluctuating electric field is created around the belt. An insulated electrode, located in the proximity of the belt, generates an induced current signal in response to the passage of the surface charges. Information about the motion of the belt can then be derived from the induced signal. Fig. 1 shows the fundamental sensing principle of the electrostatic sensor.

\section{B. Finite-Element Modeling}

According to the superposition principle of electrostatic field, the total induced charge on the electrode is equal to the

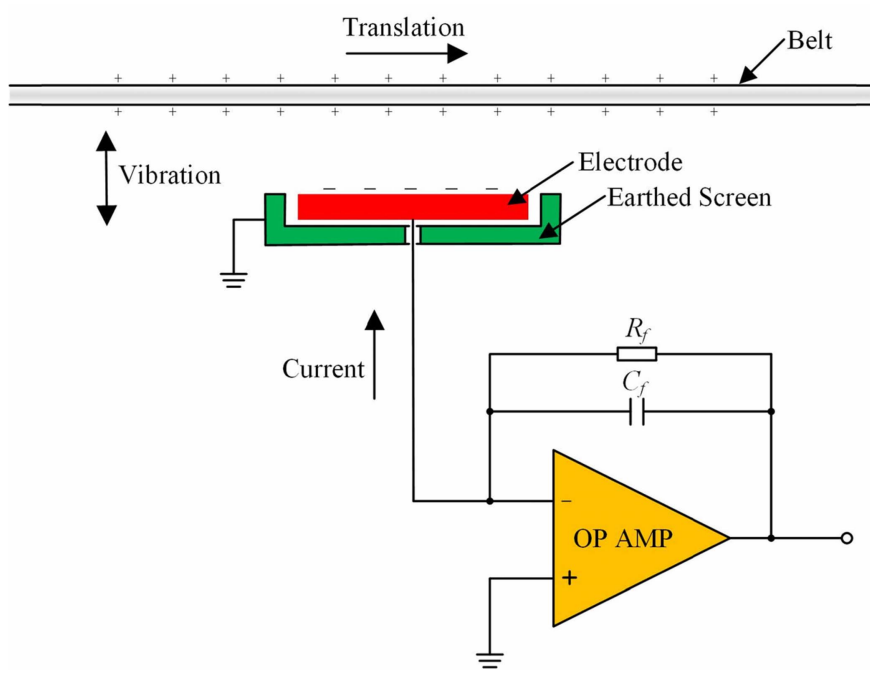

Fig. 1. Fundamental sensing principle of the electrostatic sensor.

sum of all charges induced by each individual charge on the belt. It is therefore reasonable to utilize a single point charge moving along the belt trajectory to study the characteristics of sensor response and motivate suitable measurement method. The electric field produced by a point charge is governed by the following Poisson equation:

$$
\nabla \cdot(\varepsilon \cdot \nabla \Phi)=-\rho
$$

where $\Phi$ is the electric potential, $\rho$ is the charge density, and $\varepsilon$ is the permittivity of the medium. After $\Phi$ is solved, the induced charge $q^{\prime}$ on the electrode with surface area $S$ can be calculated through surface integration of the charge density

$$
q^{\prime}=\int_{S} \varepsilon \cdot \nabla \Phi d s
$$

Analytical solutions to (1) can only be found for a very limited number of simple cases, and it is often necessary to resort to numerical methods. In this paper, a finite-element model of the electrostatic sensor is built using the commercial software package COMSOL Multiphysics. The electrode is modeled as a thin copper strip with dimensions of $20-\mathrm{mm}$ length and $3-\mathrm{mm}$ width. The point charge is a nylon sphere with negligible size. The medium of the computational region is air with a dielectric constant of 1 . The potential of the electrode is set as zero, and the boundary of the model domain is set as zero charge. The point charge carries excess electrons (negatively charged) of $1 \mu \mathrm{C}$ and is located $6 \mathrm{~mm}$ above the center of the electrode. Fig. 2 shows the simulated surface charge density on the electrode. As can be seen, the middle and the boundary areas are populated with more charges than the other areas. By surface integration, the total induced charge on the electrode is calculated as $0.0668 \mu \mathrm{C}$.

\section{Characteristics of Sensor Response}

The sensor response is simulated by letting the point charge move sinusoidally through the sensing volume of the electrode. The parametric sweep function of the software is used, and the longitudinal distance between the center of the electrode and the point charge is incremented by $0.2 \mathrm{~mm}$ in each step. 


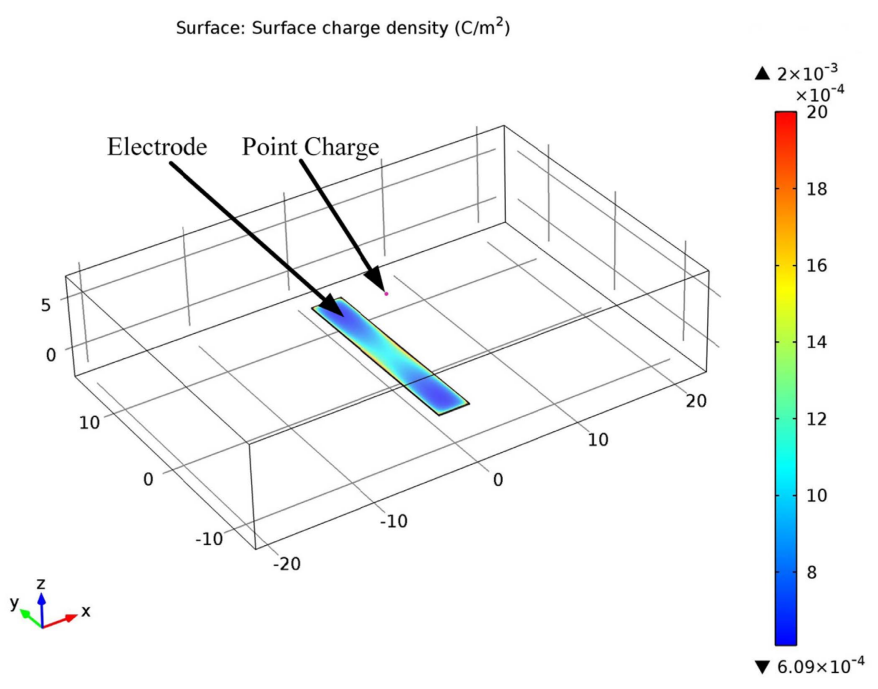

Fig. 2. Surface charge density on the electrode. (The wireframe box indicates the computational region of the finite-element model.)

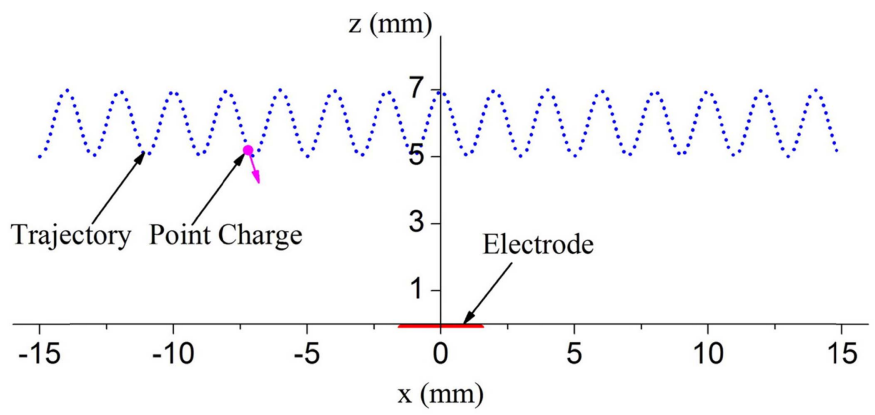

(a)

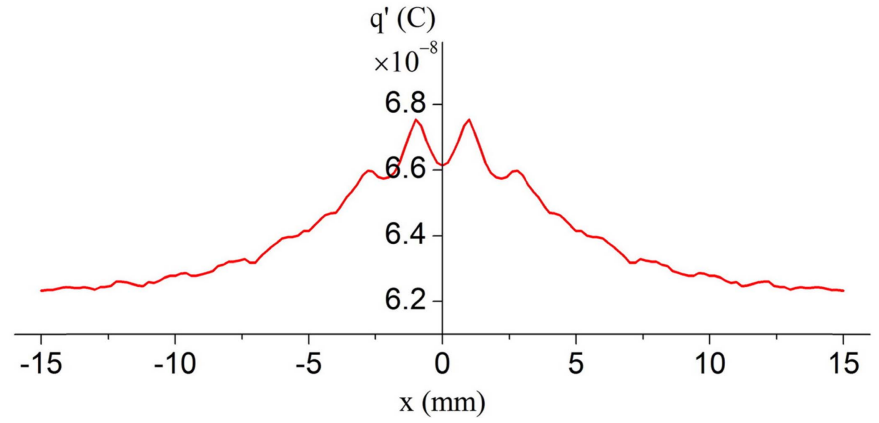

(b)

Fig. 3. Induced charge on the electrode for a sinusoidal trajectory. (a) Trajectory of the point charge. (b) Induced charge on the electrode.

Fig. 3 shows the induced charge on the electrode when the trajectory of the point charge is described by $z=6+\cos (\pi x)$ and $y=0$, and the center of the electrode is located at the origin of the coordinate system. As can be seen, when the point charge is far away from the electrode, the induced charge increases almost monotonically as the point charge approaches the electrode in the longitudinal direction. The transverse vibration of the point charge leads to small fluctuations of the induced charge. However, when the point charge is close to the electrode, the induced charge is dominated by the transverse distance of the point charge, and similar vibration modes, albeit antiphasing, are observed between the trajectory and the induced charge.
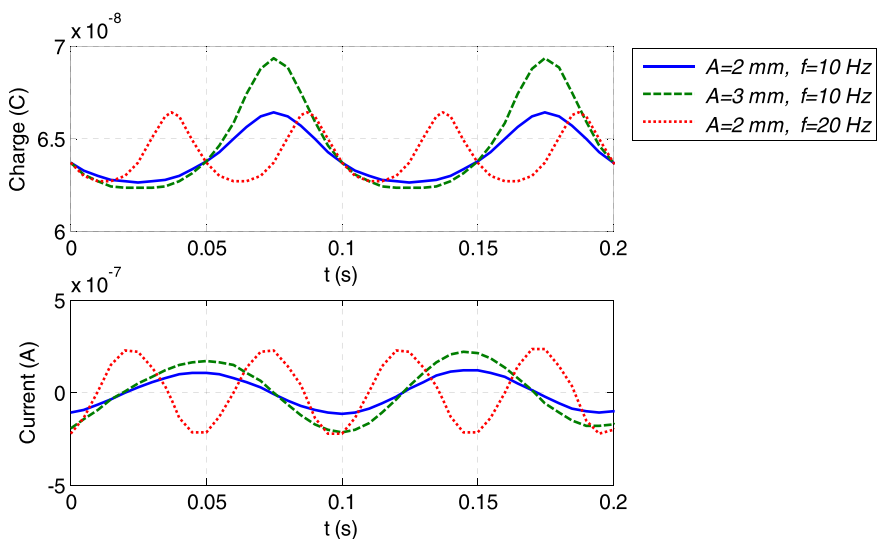

Fig. 4. Induced charge and current output for different vibration amplitudes and frequencies.

In practice, an enormous number of point charges are continuously distributed on the belt surface. If the charge distribution was perfectly uniform and the belt did not vibrate at all, the induced charge on the electrode would remain constant and there would be no current flowing through the electrode. Early research has shown that two identical electrodes placed in parallel in the belt traveling direction generate two similar signals with a time delay between them [23]. The fluctuation of the sensor signal, which is the rate of change in the total induced charge, reflects that the charge distribution on the belt surface is nonuniform, whereas the signal similarity can be explained by the relatively constant charge density on the belt. Because the nonuniformity of charge distribution along the belt is not strong, the effect of transverse vibration of nearby charges on the variation of induced charge is more significant. It is therefore hypothesized that the fluctuation of the sensor signal is dominated by the transverse belt vibration, which will be verified by experimental results (Section IV).

To further elucidate how the sensor signal is related to the belt vibration, the point charge is set to vibrate harmonically in the normal direction of the electrode surface. Suppose the trajectory of the point charge is given by $z=6+A \sin (2 \pi f t)$, where $A$ denotes the vibration amplitude and $f$ the vibration frequency. The induced charge and current output for different vibration amplitudes and frequencies are plotted in Fig. 4. As illustrated, the electrode generates more current for a larger vibration amplitude, under which a longer excursion distance is covered and the variation of the induced charge is stronger. It is also observed that the amplitude of the alternating current output increases with the vibration frequency. If the variation of the induced charge can be approximated using a sinusoidal function, it is easy to understand from a mathematical point of view that the amplitude of its time derivative (the current) is proportional to the frequency.

It is evident in Fig. 4 that the induced charge varies asymmetrically about the value when the point charge is at the center position. As a result, the current output is also asymmetrical about the zero line and in time. This effect is caused by the nonuniform spatial sensitivity of the electrostatic sensor, which is defined as the ratio of the induced charge to the source charge [25]. Fig. 5 shows the spatial sensitivity 


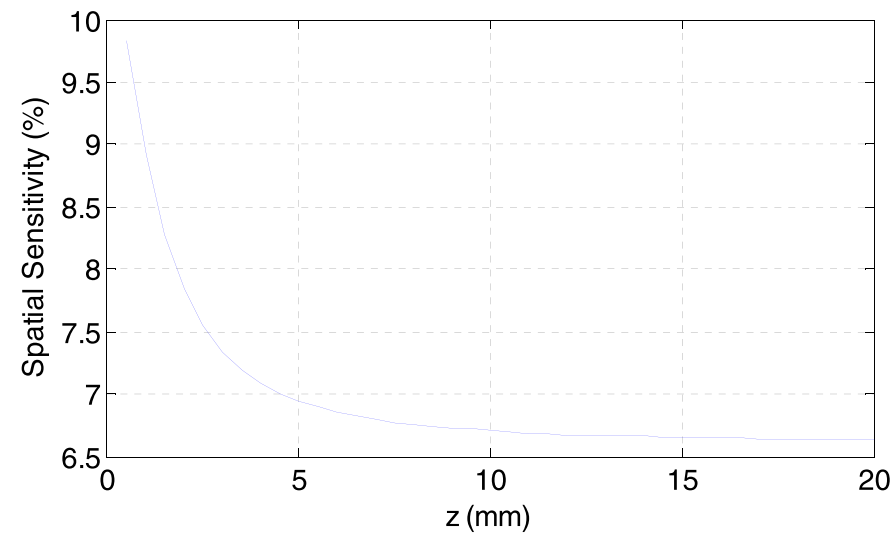

Fig. 5. Spatial sensitivity along the $z$-axis.

of the electrostatic sensor along the $z$-axis. As can be seen, the spatial sensitivity decreases nonlinearly along the $z$-axis, thus explaining the asymmetrical characteristics of the induced charge and current output. Since the spatial sensitivity is more uniform at further locations, it is advantageous to place the electrode relatively further away from the belt for a more linear relationship between the signal amplitude and the vibration displacement. However, the electrode should not be placed too far away to maintain minimum sensitivity for the measurement.

\section{Measurement Method and Sensor Design}

\section{A. Sensing Arrangement}

Measurement of the belt speed can be achieved with two identical strip-shaped electrodes placed in parallel in the belt traveling direction with a known spacing between them. The transit time taken by the belt to move from the upstream electrode to the downstream one is determined with a crosscorrelation signal processing algorithm [23]. The transverse vibration of the belt at some location along the belt traveling direction can be measured with a single electrode. Vibration parameters, such as vibration amplitude and frequency, can be derived from the fluctuation of the sensor signal. In the present research, an array of electrostatic sensors is combined with cross-correlation and data fusion algorithms to obtain accurate and reliable measurements of belt speed and vibration. Fig. 6 shows the sensing arrangement of the measurement system. Six electrodes are arranged in a $2 \times 3$ matrix. Three electrodes in a row are positioned above the belt, while the remaining three below the belt. Three separate speed measurements are obtained for each row of electrodes by permutation of the signals, while the two electrodes in a column generate a vibration measurement.

\section{B. Measurement Algorithm}

Let $s_{i}(k)$ denote the $i$ th discretized zero-mean electrostatic sensor signal, then the normalized cross-correlation function between $s_{i}(k)$ and $s_{j}(k)$ is expressed as

$$
r_{i j}(m)=\frac{\sum_{k=1}^{N-M} s_{i}(k) s_{j}(k+m)}{\sqrt{\sum_{k=1}^{N-M} s_{i}^{2}(k)} \sqrt{\sum_{k=1}^{N-M} s_{j}^{2}(k)}}
$$

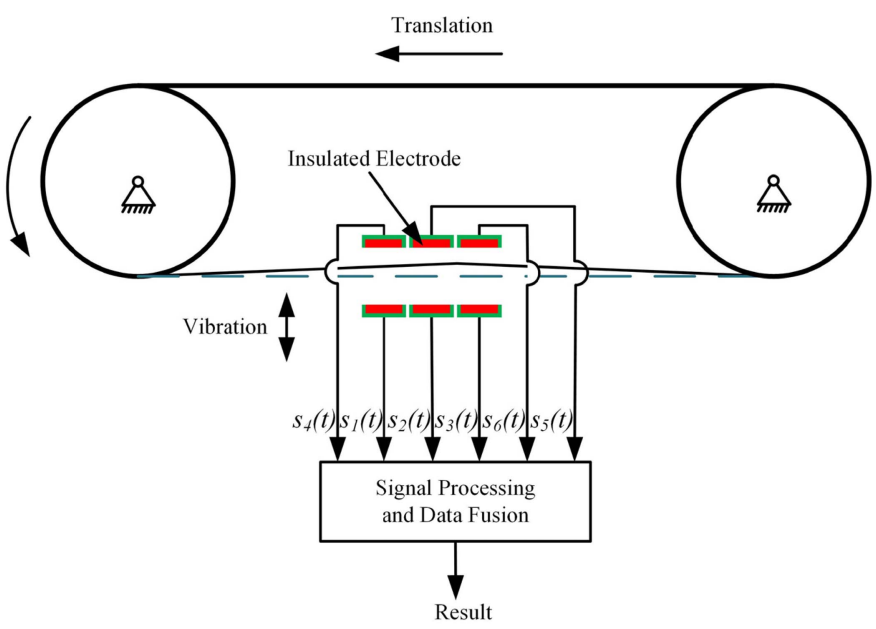

Fig. 6. Sensing arrangement of the measurement system.

where $m=0,1, \ldots, M$ is the number of delayed data points and $N$ is the length of the sample set. The location of the dominant peak in $r_{i j}(m)$ determines the transit time $\tau_{i j}$. Then the belt speed $v_{i j}$ can be calculated as

$$
v_{i j}=\frac{L_{i j}}{\tau_{i j}}
$$

where $L_{i j}$ is the center-to-center spacing between the $i$ th and the $j$ th electrodes. The value of the dominant peak $R_{i j}$, called correlation coefficient, reflects the similarity of the two signals. Since a higher $R_{i j}$ implies normally a more accurate speed measurement, it is used as the weight for $v_{i j}$ in the data fusion algorithm through linear combination. As per the belt traveling direction and the numbering of the electrodes shown in Fig. 6, the average belt speed is computed as

$$
\bar{v}=\frac{R_{12} v_{12}+R_{13} v_{13}+R_{23} v_{23}+R_{45} v_{45}+R_{46} v_{46}+R_{56} v_{56}}{R_{12}+R_{13}+R_{23}+R_{45}+R_{46}+R_{56}} .
$$

According to the simulation results, the amount of induced charge is inversely related to the distance between the belt and the electrode. The presented sensing arrangement (Fig. 6) determines that an increase in the induced charge on one electrode due to belt approaching in the transverse direction corresponds to a decrease on the other electrode in the same column, thus leading to two current signals with opposite trends. The absolute vibration displacement of the belt cannot be measured using the electrostatic sensors due to the fact that the charges carried on the belt depend on a wide range of factors including belt speed, temperature, and humidity. However, the vibration displacement can be quantitatively characterized using the fluctuation amplitude of the sensor signal. Taking into consideration the influence of the vibration frequency on the amplitude of the sensor signal, the frequency spectrum is normalized according to the following equation in order to obtain a more accurate characterization of the vibration displacement:

$$
S_{i, \text { norm }}(\omega)=\frac{\left|S_{i}(\omega)\right|}{\omega}
$$




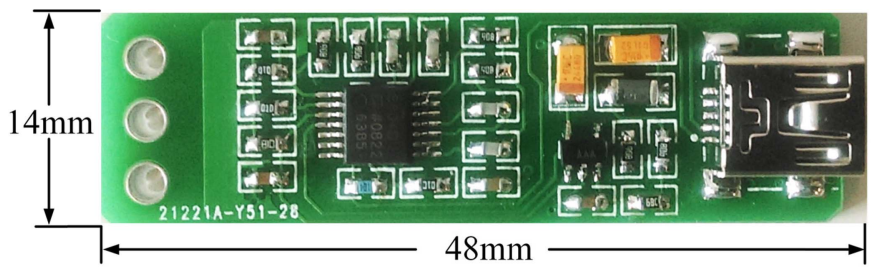

(a)

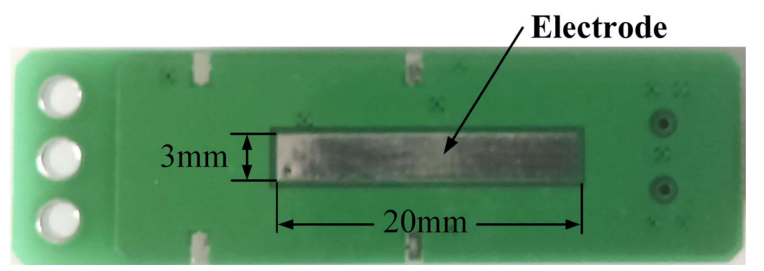

(b)

Fig. 7. Electrostatic sensor used in this paper. (a) Top layer. (b) Bottom layer.

where $S_{i}(\omega)$ represents the Fourier transform of the sensor signal $s_{i}(t)$.

The sensor signals from the pair of sensors in the same column are expected to have the same frequency spectrum. If the distances between the belt and the two corresponding electrodes are equal, then the frequency components of the two signals have the same amplitude. In order to enhance the measurement reliability, the belt vibration is characterized by averaging the normalized amplitude spectra of the signals

$$
S_{i j, \text { norm }}(\omega)=\frac{S_{i, \text { norm }}(\omega)+S_{j, \text { norm }}(\omega)}{2}
$$

where $|i-j|=3$.

\section{Sensor Design}

The electrostatic sensor is designed and fabricated based on a double-sided printed circuit board (PCB). As shown in Fig. 7, the electrode is a 3-mm wide tin-plated copper strip embedded in the bottom layer of the PCB. The area around the electrode is filled with earthed copper in order to reduce the influence of external electromagnetic interferences. On the top layer of the PCB is the signal conditioning circuit. The close placement of the signal conditioning circuit to the electrode eliminates the noise due to cable transmission. The current signal from the electrode is first converted into a proportional voltage signal using an $I / V$ converter. Because the vibration frequency of the belt is below a few $1000 \mathrm{~Hz}$, the second-order low-pass filter with a cutoff frequency of $20 \mathrm{kHz}$ is used to eliminate high-frequency noises. Then the bipolar signal is further amplified and level shifted to match the input range of a single-supply analog-to-digital converter. A DSP-based data acquisition module simultaneously samples the six electrostatic sensor signals and performs on-board data processing. Fig. 8 shows the signal conditioning stages of the sensor electronics.

\section{EXPERIMENTAL RESUlTS AND DisCUSSION}

\section{A. Test Rig}

A laboratory-scale test rig, as shown in Fig. 9, was designed and constructed for the experimental assessment of the

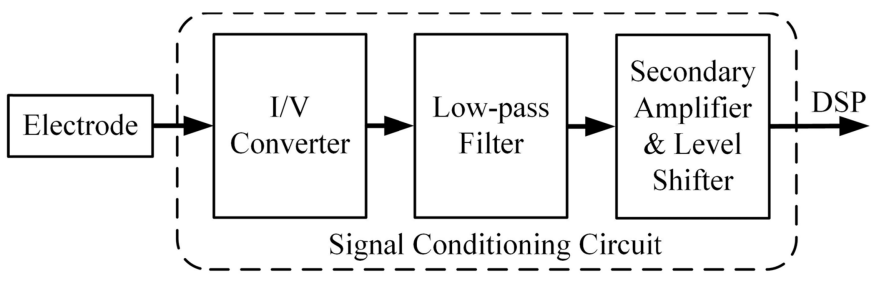

Fig. 8. Signal conditioning stages of the sensor electronics.

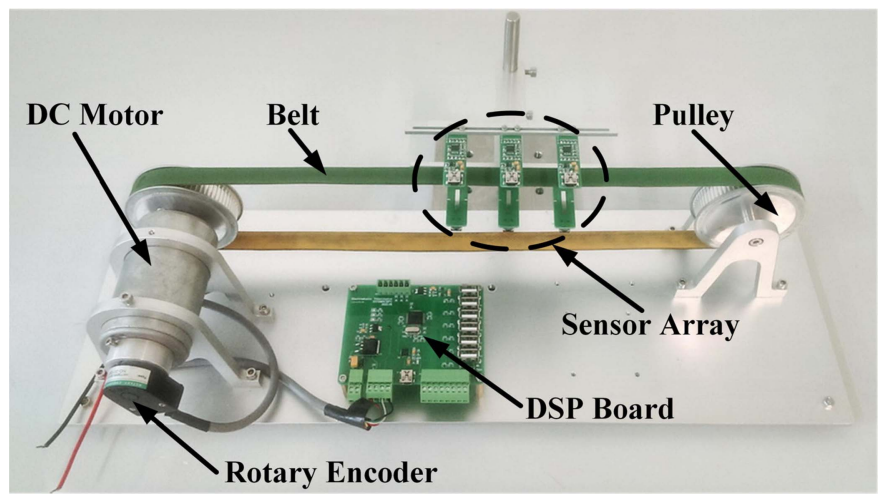

Fig. 9. Test rig for the measurement of belt speed and vibration.

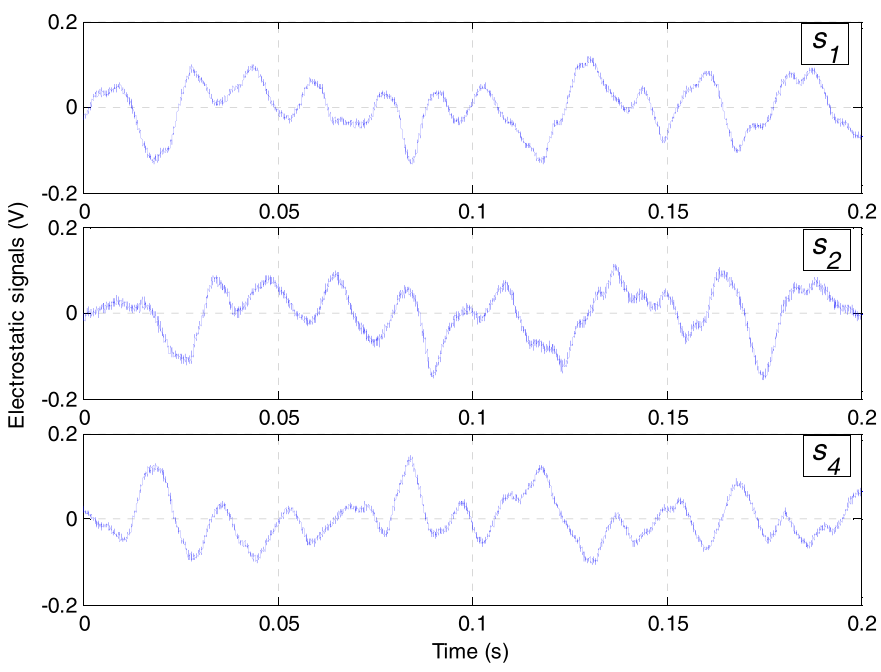

Fig. 10. Typical electrostatic signals from sensors 1, 2, and 4 .

measurement system. A flat nylon-type belt is wrapped around two fixed pulleys with an equal diameter. One of the pulleys is driven by a dc motor, the speed of which is adjusted by regulating the supply voltage. Since there is no load on the driven pulley, a resting tension force of a few newtons is adequate to prevent slippage between the belt and the pulley [26]. Therefore, the rotational speed detected using a photoelectric rotary encoder is used to derive the traveling speed of the reference belt. The sensor array is mounted on a supporting frame that allows the distance between the belt and the electrodes to be adjusted.

\section{B. Sensor Signals}

Fig. 10 shows the typical electrostatic sensor signals when the belt traveling speed is $4.3 \mathrm{~m} / \mathrm{s}$, the spacing between 


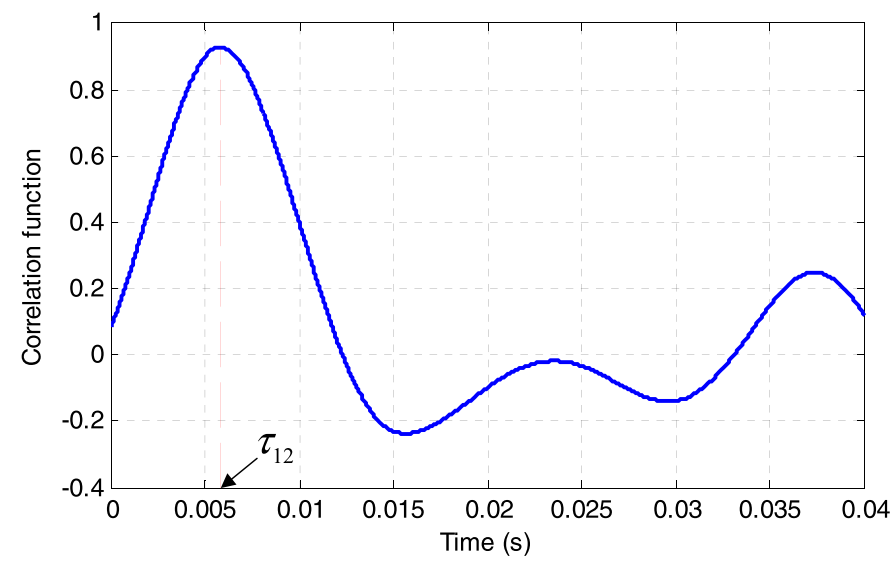

Fig. 11. Cross-correlation function between signals $s_{1}$ and $s_{2}$ in Fig. 10.

two adjacent electrodes in a row is $25 \mathrm{~mm}$, and the distance between the electrode and the belt is $12 \mathrm{~mm}$. It is clear that signal $s_{2}$ is similar to $s_{1}$ in waveform apart from the short delay in time between them. A typical correlation function between $s_{1}$ and $s_{2}$ is plotted in Fig. 11. The highest correlation coefficient reaches 0.92 , which suggests that the measured speed is adequately reliable. A variety of factors, such as the belt speed, the spacing between the electrodes, the distance between the belt and the electrode, and the environmental conditions, all affect the correlation coefficient. In this paper, if a pair of signals gives a correlation coefficient less than 0.6 , the measured speed will not be included in the data fusion algorithm.

As shown in Fig. 10, signals $s_{1}$ and $s_{4}$ exhibit nearly antiphasing oscillatory behaviors, therefore the statement that the signal fluctuations are primarily caused by the transverse belt vibrations can be validated. The normalized amplitude spectra of signals $s_{1}$ and $s_{4}$ in Fig. 10, but of a longer period of time $(2.0 \mathrm{~s})$, are plotted in Fig. 12, where the spectral peaks are marked with small circles. It is clearly seen that the two spectra resemble each other, with the same spectral peaks that correspond to vibration modes occurring at several distinct frequencies below $100 \mathrm{~Hz}$. It is believed that the spectral peaks at exactly $50 \mathrm{~Hz}$ are attributed to the power line interference.

The belt vibration leads to a traveling wave that propagates along the length of the belt, therefore the sensors in different columns experience different phases of the traveling wave. The

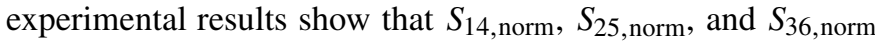
have nearly the same dominant frequencies and corresponding amplitudes. However, the phase shifts can be easily observed from the signal waveforms (Fig. 10). The magnitude of phase shifts depends on the spacing of the electrodes as well as the wavelength of the traveling wave, which is determined by the belt dynamics.

\section{Speed Measurement Results}

The accuracy of the system for belt speed measurement was evaluated by varying the reference belt speed from 2 to $10 \mathrm{~m} / \mathrm{s}$ and the distance between the electrodes and the belt from 4 to $20 \mathrm{~mm}$. Fig. 13 shows the relative error between the measured speed and the reference speed. The measured speed

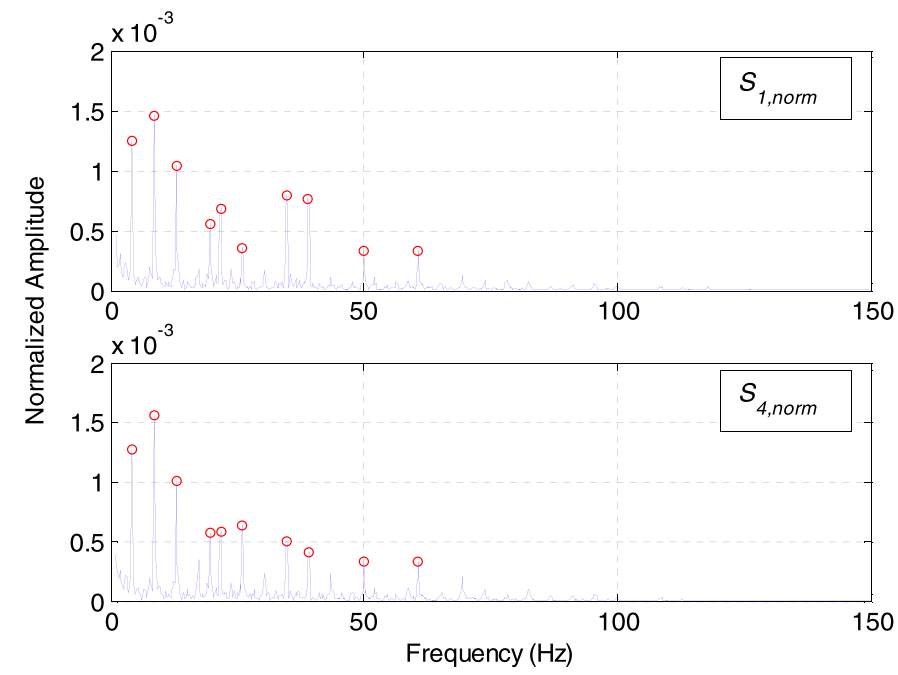

Fig. 12. Normalized amplitude spectra of typical electrostatic signals.

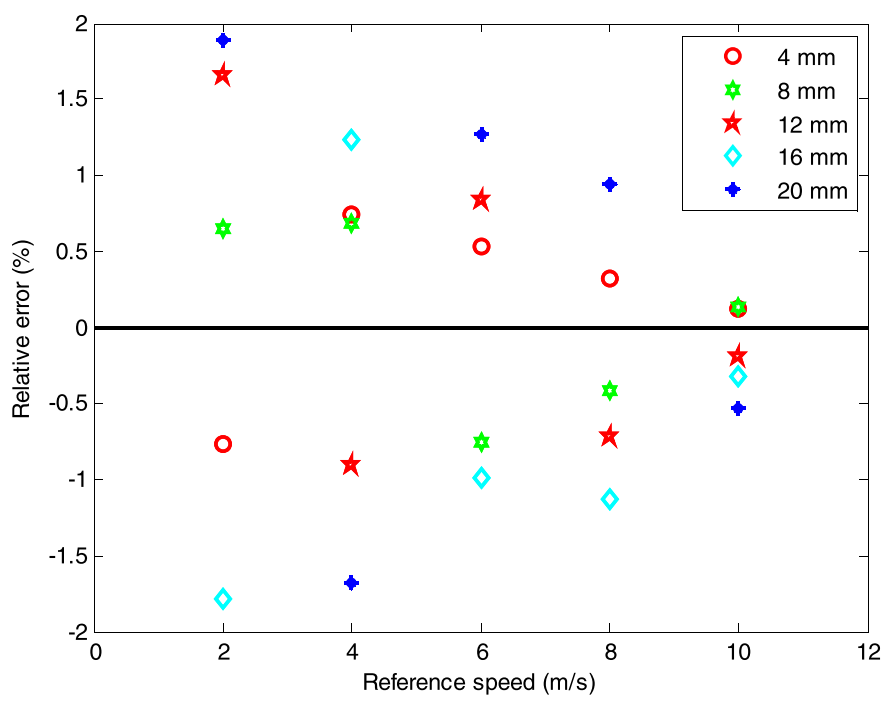

Fig. 13. Relative error of the measured speed.

is the average value of 20 measurements with normalized standard deviation less than $0.8 \%$. Throughout the speed measurement range, the relative error of the measured speed is no greater than $\pm 2 \%$. The relative error becomes smaller as the belt runs faster, which is attributed to the increased charge on the belt surface and stronger belt vibration at higher speeds. The shorter distance between the electrodes and the belt surface also yields more accurate measurements because of the stronger signals from the sensors. However, the electrodes should not be excessively close to the belt in order to avoid the potential contact between the electrodes and the belt, which could damage the sensors and give rise to erroneous signals.

The performance improvement due to the use of the sensor array and the data fusion algorithm was validated by comparing the six raw speeds each obtained with two sensors and the fused speed. Twenty sets of data were collected at different belt speeds, respectively. As shown in Fig. 14, the normalized standard deviations of the raw speeds are greater than that of the fused speed, suggesting that the fused speed is more stable and accurate than the individual measurements. In general, the normalized standard deviations of $v_{13}$ and $v_{46}$ are larger 


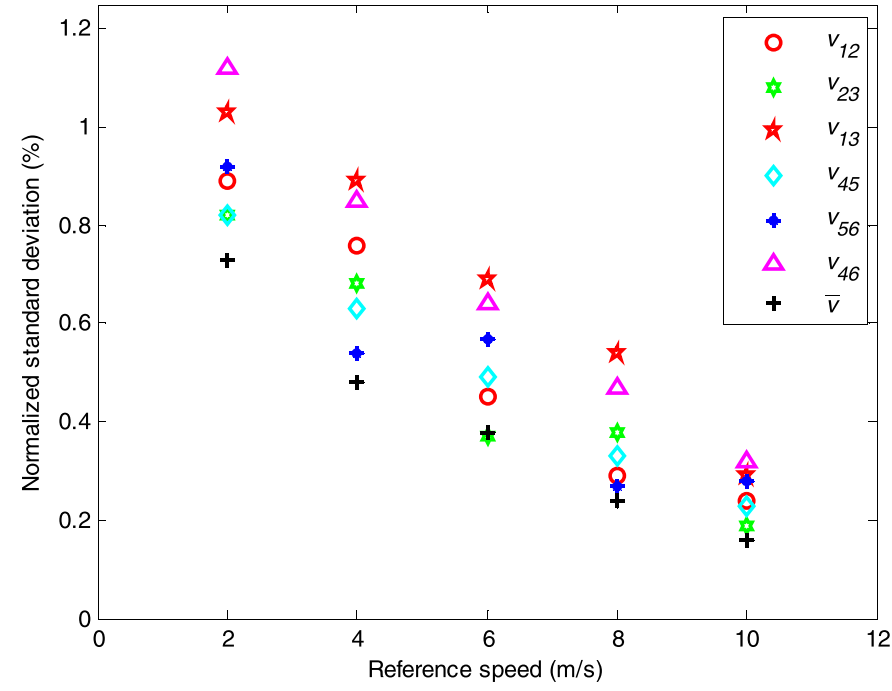

Fig. 14. Normalized standard deviation of the measured speed.

than that of the other combinations because the longer spacing between the electrodes results in poorer similarity between the signals and hence lower correlation coefficient. As expected, the normalized standard deviations of the individual speeds are reduced as the belt speed increases, thus leading to a fused speed that is more repeatable.

The uncertainty of speed measurement results arises from several possible sources. The first one is the stochastic nature of the electrostatic signals. The charges appear on the belt due to continuous motion of the belt and vanish with the elapse of time, reaching a dynamic balance for a given operating condition. The change of charge distribution on the belt leads to similar but not identical signals from adjacent sensors, introducing some degree of uncertainty to the cross-correlationbased speed measurement. The second one is concerned with the accuracy in the measured transit time. Because the analog signals are digitized for numerical computation, it is unlikely that the real transit time is located at the exact discrete points of the cross-correlation function. The use of a higher sampling rate and/or an adequate interpolation algorithm to fit the points around the peak will reduce this uncertainty, but the cost is higher due to the increased computation load. The third source originates from the vibration of the sensors themselves. The mechanical connection to the machine makes the sensors vibrate in both the axial and transverse directions of the belt. The axial vibration of the sensors increases or decreases the speed measurement results, depending on the vibration direction during sampling. The final source of uncertainty lies in mechanical manufacturing and assembly errors. The dimension of the electrode may not follow the design exactly, and distance inaccuracy exists in the spacing of the electrodes. These systematic errors can be reduced through compensation or calibration.

\section{Vibration Measurement Results}

An axially moving belt vibrates transversely at natural frequencies determined by belt mass, tension, speed, span length, and viscoelastic stiffness [27], whereas a variety of installation and belt imperfections, such as shaft misalignment, pulley eccentricity, belt wear, and imbalance, cause vibration at frequencies other than the natural ones. For instance, problems due to shafts or pulleys (misalignment, eccentricity, etc.) result in vibration at the rotational frequency of the component. Stretched or worn belts, on the other hand, give rise to vibration at harmonics of the belt pass frequency [28]. In order to assess the validity of the vibration measurement results, a stretched belt is used to generate vibration with known frequencies. The belt pass frequency is given by

$$
f=\frac{v}{L}
$$

where $v$ is the belt running speed and $L$ is the belt length.

Fig. 15 shows the fused vibration spectra at different belt running speeds. As indicated in Fig. 15, the belt vibrates at frequencies that are integer (whole number) multiple of the fundamental frequency. Since the length of the stretched belt is $0.99 \mathrm{~m}$, the belt pass frequency calculated using (8) is almost identical to the fundamental vibration frequency, thus validating the effectiveness of the technique. It is evident that the second-order harmonic is the highest peak in Fig. 15(b) and (c), which is the typical symptom of using stretched belts. It is also shown that as the belt runs faster, more vibration modes are provoked, especially at higher frequencies. The spectrum peaks become stronger at a higher speed, which suggests that the vibration displacement becomes larger. However, the increased transverse deflection is not the sole reason for the elevated signal strength, because more charges are accumulated on the belt at a higher speed.

\section{E. Discussion}

Given that the methods available for the measurement of belt speed and vibration are very limited, the electrostatic sensing technique represents an appealing solution to the current measurement problem, with the virtue of non-contact and simultaneous measurement, low cost, simple structure, and easy installation. However, this technique has several inherent limitations that might be resolved in the future. First, the amount of induced charge on the electrode is susceptible to environmental and operating conditions, which makes it impossible to measure the absolute vibration displacement. Nevertheless, the location of the spectral peaks and their relative magnitude remain fixed for a specific operating condition. Such information can still provide valuable status indication for online diagnosis and prognosis of the belt drive system. The changes in the environmental and operating conditions may make the charge on the belt vary over orders of magnitude. In this regard, it would be advantageous to use a programmable gain amplifier for online adjustment of the resulting signal strength. Second, it is clearly seen in Fig. 15 that the sensor signal contains the $50-\mathrm{Hz}$ power line noise, the magnitude of which is independent of the belt running speed. As the belt speed increases and the sensor signal becomes stronger, the signal-to-noise ratio is improved, which leads to enhanced measurement accuracy. The working principle of electrostatic induction determines that it is difficult to 


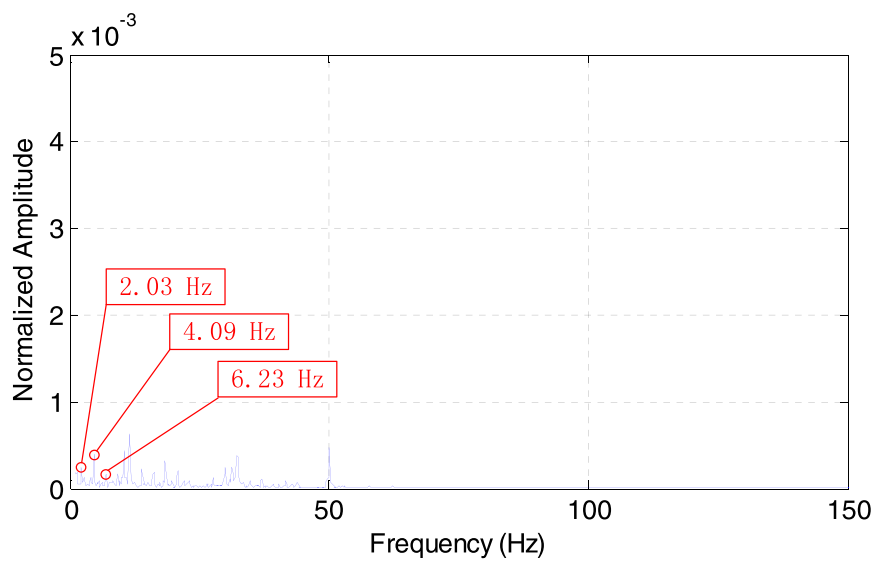

(a)

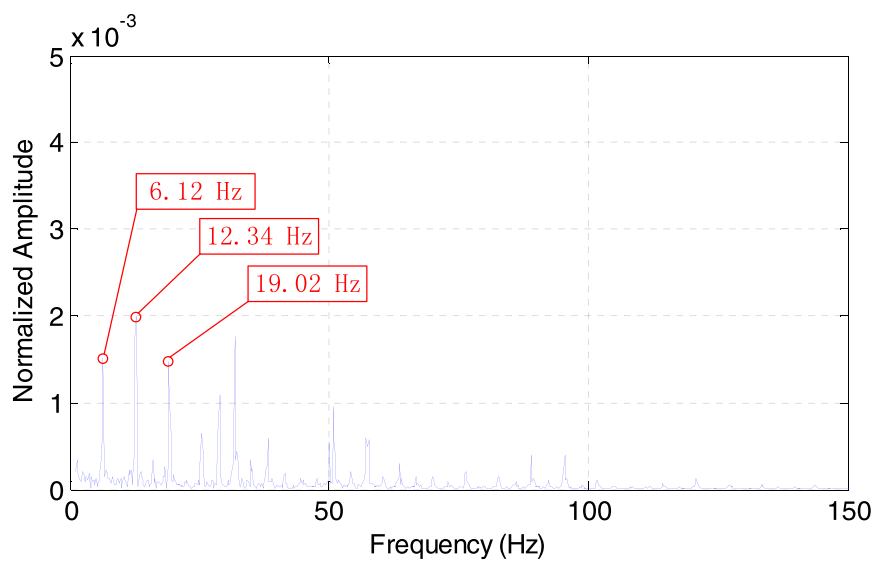

(b)

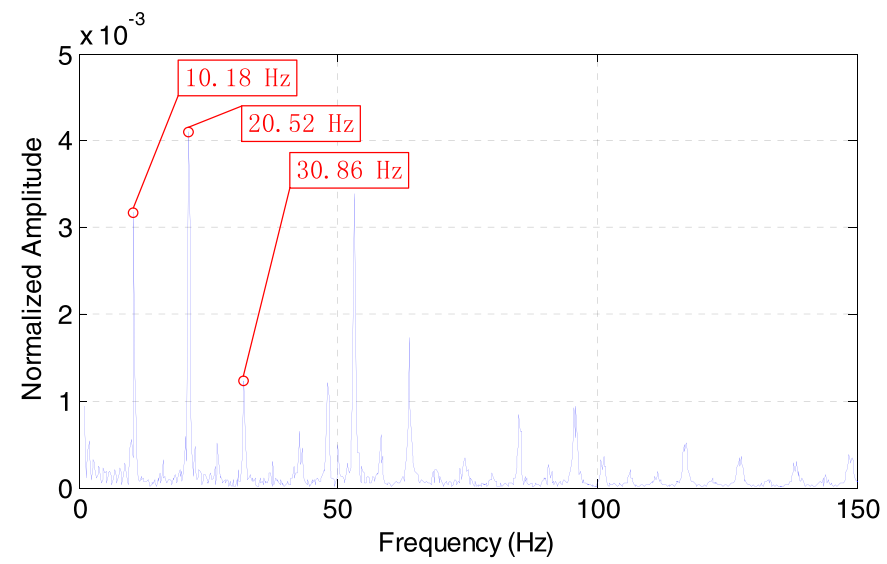

(c)

Fig. 15. Normalized spectra of the electrostatic signals at different belt speeds. (a) $2 \mathrm{~m} / \mathrm{s}$. (b) $6 \mathrm{~m} / \mathrm{s}$. (c) $10 \mathrm{~m} / \mathrm{s}$.

completely shield the electrode using a metal screen to reject external electromagnetic interferences. It is also not possible to filter out this noise using a notch filter, because its frequency is within the range of vibration. For the present study conducted in laboratory conditions, the noise is not a major concern as its frequency and magnitude are definite and known. However, the application of the technique in an industrial environment with various sources of electromagnetic interference may be problematic. A practical technique to avoid electromagnetic interferences should be developed. Third, the experimental work was undertaken on a two-pulley belt drive system at steady rotational speed and without load on the driven pulley. The belt vibration dynamics is relatively simple and the performance of the system in monitoring more erratic belt vibrations remains to be investigated. A direct comparison of the measurement results with another reference system such as a laser vibrometer may provide a deeper insight into the pros and cons of the technique.

\section{CONCLUSION}

In this paper, a novel measurement system based on an electrostatic sensor array and data fusion techniques has been designed and implemented for simultaneous measurement of belt speed and vibration. The response of a strip-shaped electrode to a point charge moving both axially and transversely has been studied through finite-element modeling that has suggested how the sensor design should be optimized and how the sensor signals should be processed to achieve more accurate and reliable measurements. Experimental investigations into the performance of the measurement system have been conducted on a custom-built test rig. The results obtained have demonstrated that the system can measure the belt speed with a relative error within $\pm 2 \%$ over the range $2-10 \mathrm{~m} / \mathrm{s}$. The accuracy and repeatability of the speed measurement results are enhanced due to the use of the sensor array and the data fusion technique. The vibration modes of the belt at different speeds have been identified using spectrum analysis technique. Higher belt speeds have caused more high-frequency vibration modes to be provoked and larger amplitude deflection produced.

Further work will focus on quantifying the effects of belt type and surface roughness on the measurement system in terms of accuracy, repeatability, and reliability under a wider range of environmental conditions. Performance assessment of the system on industrial belt drives with complex dynamic behaviors will also be carried out in the near future.

\section{ACKNOWLEDGMENT}

The authors would like to thank the IEEE Instrumentation and Measurement Society for offering an IEEE Graduate Fellowship Award in relation to the research reported in this paper.

\section{REFERENCES}

[1] M. J. Leamy and T. M. Wasfy, "Transient and steady-state dynamic finite element modeling of belt-drives," J. Dyn. Syst., Meas., Control, vol. 124, no. 4, pp. 575-581, 2002.

[2] M. Musselman and D. Djurdjanovic, "Tension monitoring in a beltdriven automated material handling system," CIRP J. Manuf. Sci. Technol., vol. 5, no. 1, pp. 67-76, 2012.

[3] L. Manin, G. Michon, D. Remond, and R. Dufour, "From transmission error measurement to pulley-belt slip determination in serpentine belt drives: Influence of tensioner and belt characteristics," Mech. Mach. Theory, vol. 44, no. 4, pp. 813-821, 2009.

[4] Y. Pang and G. Lodewijks, "A novel embedded conductive detection system for intelligent conveyor belt monitoring," in Proc. IEEE Int. Conf. Service Oper. Logistics, Inform., Shanghai, China, Jun. 2006, pp. 803-808.

[5] S. J. Arif, M. S. J. Asghar, and A. Sarwar, "Measurement of speed and calibration of tachometers using rotating magnetic field," IEEE Trans. Instrum. Meas., vol. 63, no. 4, pp. 848-858, Apr. 2014. 
[6] D. Garcia-Vizcaino, F. Dios, J. Recolons, A. Rodríguez, and A. Comeron, "One-wavelength two-component laser Doppler velocimeter system for surface displacement monitoring," Opt. Eng., vol. 47, no. 12, p. 123606, 2009.

[7] T. O. H. Charrett, S. W. James, and R. P. Tatam, "Optical fibre laser velocimetry: A review," Meas. Sci. Technol., vol. 23, no. 3, p. 032001, 2012.

[8] M. Shin, Z. Zhao, P. DeBitetto, and R. D. White, "Micromachined ultrasonic Doppler velocity sensor using nickel on glass transducers," Sens. Actuators A, Phys., vol. 208, pp. 37-49, Feb. 2014.

[9] P. Descamps, J. Vindevoghel, F. Bouazza, and S. Sawsan, "Microwave Doppler sensors for terrestrial transportation applications," IEEE Trans. Veh. Technol., vol. 46, no. 1, pp. 220-228, Feb. 1997.

[10] J. M. Lopera, M. J. Prieto, F. F. Linera, G. Vecino, and J. A. González, "A new speed measurement system," IEEE Ind. Appl. Mag., vol. 11, no. 6, pp. 44-51, Nov./Dec. 2005.

[11] E. M. Deeley and P. Kontopoulous, "Strip speed measurement using distributed infra-red detectors," IEE Proc. D, Control Theory Appl., vol. 138, no. 4, pp. 395-399, Jul. 1991.

[12] A. Agnani, M. Martarelli, and E. P. Tomasini, "V-belt transverse vibration measurement by means of laser Doppler vibrometry," Proc. SPIE, vol. 7098, p. 709819, Jun. 2008.

[13] C. Yang et al., "A multi-point laser Doppler vibrometer with fiber-based configuration," Rev. Sci. Instrum., vol. 84, no. 12, p. 121702, 2013.

[14] H. Chaurasiya, "Recent trends of measurement and development of vibration sensors," Int. J. Comput. Sci. Issues, vol. 9, no. 4, pp. 353-358, 2012.

[15] J. R. Coombes and Y. Yan, "Experimental investigations into the flow characteristics of pneumatically conveyed biomass particles using an electrostatic sensor array," Fuel, vol. 151, pp. 11-20, Jul. 2015.

[16] X. Qian, X. Huang, H. Yonghui, and Y. Yan, "Pulverized coal flow metering on a full-scale power plant using electrostatic sensor arrays," Flow Meas. Instrum., vol. 40, pp. 185-191, Dec. 2014.

[17] W. Zhang, Y. Cheng, C. Wang, W. Yang, and C.-H. Wang, "Investigation on hydrodynamics of triple-bed combined circulating fluidized bed using electrostatic sensor and electrical capacitance tomography," Ind. Eng. Chem. Res., vol. 52, no. 32, pp. 11198-11207, 2013.

[18] H. Zhou et al., "Investigation of two-phase flow mixing mechanism of a swirl burner using an electrostatic sensor array system," Flow Meas. Instrum., vol. 32, pp. 14-26, Aug. 2013.

[19] T. Addabbo, A. Fort, R. Garbin, M. Mugnaini, S. Rocchi, and V. Vignoli, "Theoretical characterization of a gas path debris detection monitoring system based on electrostatic sensors and charge amplifiers," Measurement, vol. 64, pp. 138-146, Mar. 2015.

[20] Z. Wen, X. Ma, and H. Zuo, "Characteristics analysis and experiment verification of electrostatic sensor for aero-engine exhaust gas monitoring," Measurement, vol. 47, pp. 633-644, Jan. 2014.

[21] P. Intra, A. Yawootti, and N. Tippayawong, "An electrostatic sensor for the continuous monitoring of particulate air pollution," Korean J. Chem. Eng., vol. 30, no. 12, pp. 2205-2212, 2013.

[22] L. Wang, Y. Yan, Y. Hu, and X. Qian, "Rotational speed measurement using single and dual electrostatic sensors," IEEE Sensors J., vol. 15, no. 3, pp. 1784-1793, Mar. 2015.

[23] Y. Yan, S. J. Rodrigues, and Z. Xie, "Non-contact strip speed measurement using electrostatic sensing and correlation signal-processing techniques," Meas. Sci. Technol., vol. 22, no. 7, p. 075103, 2011.

[24] Y. Hu, L. Wang, X. Wang, X. Qian, and Y. Yan, "Simultaneous measurement of conveyor belt speed and vibration using an electrostatic sensor array," in Proc. IEEE Int. Instrum. Meas. Technol. Conf., Pisa, Italy, May 2015, pp. 757-761.

[25] Y. Yan, B. Byrne, S. Woodhead, and J. Coulthard, "Velocity measurement of pneumatically conveyed solids using electrodynamic sensors," Meas. Sci. Technol., vol. 6, no. 5, pp. 515-537, 1995.

[26] R. S. Khurmi and J. K. Gupta, A Textbook of Machine Design, 14th ed. New Delhi, India: Eurasia Publishing House (Pvt.) Ltd., 2005.

[27] U. Lee and H. Oh, "Dynamics of an axially moving viscoelastic beam subject to axial tension," Int. J. Solids Struct., vol. 42, no. 8, pp. 2381-2398, 2005.

[28] R. K. Mobley, Vibration Fundamentals. Boston, MA, USA: ButterworthHeinemann, 1999.

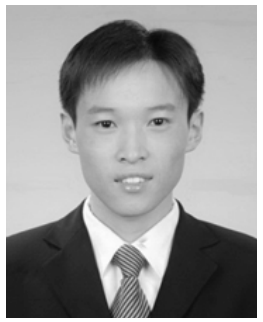

Yonghui Hu received the B.Eng. degree in automation from the Beijing Institute of Technology, Beijing, China, in 2004, and the Ph.D. degree in dynamics and control from Peking University, Beijing, in 2009.

He was a Post-Doctoral Research Fellow with Beihang University, Beijing, from 2010 to 2012 $\mathrm{He}$ is currently a Lecturer with the School of Control and Computer Engineering, North China Electric Power University, Beijing. His current research interests include measurement of multiphase flow and condition monitoring of mechanical systems.

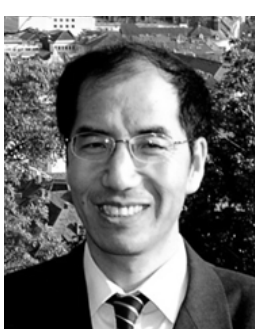

Yong Yan (M'04-SM'04-F'11) received the B.Eng. and M.Sc. degrees in instrumentation and contro engineering from Tsinghua University, Beijing, China, in 1985 and 1988, respectively, and the $\mathrm{Ph} . \mathrm{D}$. degree in flow measurement and instrumentation from the University of Teesside, Middlesbrough, U.K., in 1992.

$\mathrm{He}$ was an Assistant Lecturer with Tsinghua University in 1988. He joined the University of Teesside in 1989, as a Research Assistant. After a short period of post-doctoral research, he was a Lecturer with the University of Teesside from 1993 to 1996, and then as a Senior Lecturer, Reader, and Professor with the University of Greenwich, Chatham, U.K., from 1996 to 2004. He is currently a Professor of Electronic Instrumentation, the Head of the Instrumentation, Control and Embedded Systems Research Group, and the Director of Research with the School of Engineering and Digital Arts, University of Kent, Canterbury, U.K. $\mathrm{He}$ has authored in excess of 300 research papers in journals and conference proceedings with an $\mathrm{H}$-index of 33 .

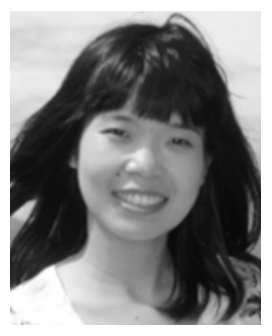

Lijuan Wang received the B.Eng. degree in computer science and technology from Qiqihar University, Qiqihar, China, in 2010, and the Ph.D. degree in measurement and automation from North China Electric Power University, Beijing, China, in 2015.

She is currently involved in a post-doctoral research in instrumentation and measurement with North China Electric Power University, Beijing. Her current research interests include electrostatic sensing, flow measurement, and digital signal processing.

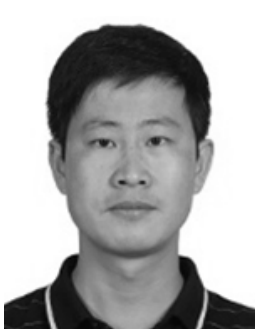

Xiangchen Qian received the B.Eng. degree in automation from the Tianjin University of Technology, Tianjin, China, in 2004, the M.Sc. degree in automatic meter and device from Tianjin University, Tianjin, in 2007, and the $\mathrm{Ph} . \mathrm{D}$. degree in electronic engineering from the University of Kent, Canterbury, U.K., in 2013.

$\mathrm{He}$ is currently a Lecturer with the School of Control and Computer Engineering, North China Electric Power University, Beijing, China. His current research interests include multiphase flow measurement techniques, development of instrumentation systems, and digital signal processing.

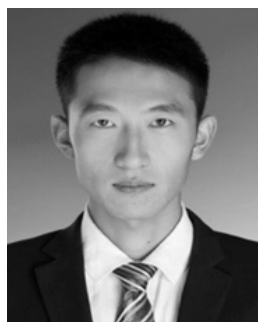

Xiaoyu Wang received the B.Eng. degree in measuring and control technology and instrument from Northeast Dianli University, Jilin City, China, in 2009, and the M.Sc. degree in instrumentation and measurement from North China Electric Power University, Beijing, China, in 2015.

$\mathrm{He}$ is currently an Engineer with China Aerospace Science and Technology Corporation, Beijing. His specialty lies in electronic circuit design and digital signal processing. 